\title{
XOR Logic Gate by Carbon/Metal Nanoinks Based on a Double-Stage Optical Kerr Gate Configuration
}

\author{
J. A. García-Merino, ${ }^{1}$ E. Feria-Reyes, ${ }^{1}$ C. Mercado-Zúñiga ${ }^{D},{ }^{2}$ M. Trejo-Valdez, ${ }^{3}$ \\ C. R. Torres-San Miguel, ${ }^{1}$ J. R. Vargas-García, ${ }^{4}$ and C. Torres-Torres $\mathbb{D i D}^{1}$ \\ ${ }^{1}$ Sección de Estudios de Posgrado e Investigación, Escuela Superior de Ingeniería Mecánica y Eléctrica Unidad Zacatenco, \\ Instituto Politécnico Nacional, 07738 Ciudad de México, Mexico \\ ${ }^{2}$ Departamento Ing. Materiales, Tecnológico de Estudios Superiores de Coacalco, 55700 Coacalco de Berriozábal, MEX, Mexico \\ ${ }^{3}$ Escuela Superior de Ingeniería Química e Industrias Extractivas, Instituto Politécnico Nacional, 07738 Ciudad de México, Mexico \\ ${ }^{4}$ Centro de Nanociencias y Micro y Nanotecnologías, Instituto Politécnico Nacional, 07738 Ciudad de México, Mexico
}

Correspondence should be addressed to C. Torres-Torres; crstorres@yahoo.com.mx

Received 26 January 2018; Accepted 20 March 2018; Published 2 May 2018

Academic Editor: Jörg Fink

Copyright (C) 2018 J. A. García-Merino et al. This is an open access article distributed under the Creative Commons Attribution License, which permits unrestricted use, distribution, and reproduction in any medium, provided the original work is properly cited.

Two-input binary exclusive-or logic operations were presented by the assistance of multiwall carbon nanotubes in a double-stage optical Kerr gate scheme with two control beams. The samples were prepared by the aerosol pyrolysis method and decorated by platinum nanoparticles using a chemical vapor deposition technique. The nanostructures were suspended in ethanol to obtain carbon/metal nanoinks with different concentrations and then randomly distributed networks integrated in thin film form were fabricated. Polarization-selectable functions were obtained in the double-stage scheme by using nanosecond third-order nonlinear optical effects at $532 \mathrm{~nm}$ wavelength exhibited by the samples. Potential applications for ultrafast identification and encryption of nonlinear optical signals were discussed.

\section{Introduction}

Hybrid nanomaterials are at the forefront of technology because they promise unique conjugate physicochemical properties [1]. The progress in the development of high precision instruments for many fields of science currently involves compound nanostructures [2]. One of the most inquired nanosystems corresponds to the carbon-based nanostructures due to their adaptive properties [3]. Particularly, carbon nanotubes (CNTs) have marked a technological impact regarding their superlative mechanical features [4]. Specifically, the multiwall carbon nanotubes (MWCNTs) are attractive in nonlinear optics due to their exceptional absorption and refractive index characteristics $[5,6]$. The cubic nonlinearities exhibited by carbon-based materials have been related to an ultrafast relaxation characterized by picosecond or subpicosecond effects [7]. Furthermore, the electrical and optical behavior exhibited by MWCNTs can be enhanced by metallic nanoparticle decoration because they increase their low free electron density [8]. In general, the development of nonlinear advanced materials is a crucial step towards the improvement of all-optical nanotechnology [9]. An important and fundamental property of metallic nanoparticles (NPs) concerns their Surface Plasmon Resonance (SPR) [10]. This property is due to a collective oscillation in the plasma of the electrons and transmitted light with a sharp selective wavelength depending on the material and the size of the NPs [11]. Moreover, the use of MWCNTs to support metallic NPs seems to be a suitable alternative for tailoring numerous functions [12, 13]. Using electrochemical deposition methods, a good control on size and densities of decorating CNTs has been accomplished [14]. Fuel cells, electronic signal modulation, photonic sensing, and nanomechanical actuators are some of the most attractive applications that can be designed by using CNT enhanced with metallic NPs [15]. In this regard, platinum presents the 
advantage of a SPR located in the ultraviolet region of the electromagnetic spectrum, and then it can be considered for high energy single photon devices [16]. Moreover, platinum decorated MWCNTs can be contemplated for developing low-dimensional platforms for photoconductive and optical circuits [17].

In order to overlap two similar materials with different effects, a double mechano-optical modulation setup based in optical Kerr gate was achieved. Functionalized thin films hybrid samples with MWCNTs decorated with different Pt concentration were placed in a cascade arrangement. In this experiment, multiphotonic phenomena have been contemplated for applications in exclusive-or logic gates to selected optical signals. With this motivation, the nonlinear optical response exhibited by randomly distributed MWCNTs networks was tailored by Pt NPs decoration in this work. A double-stage optical Kerr gate system was employed to control the positive and negative optical phase modification related to nanosecond pulses. An XOR logic function was proposed and the possibility of encrypting information by rotating nanostructures was analyzed.

\section{Materials and Methods}

2.1. Samples Synthesis. Two kinds of MWCNTs samples were prepared by an effective method based on the catalytic decomposition of metalorganic precursors [18]. The nanotubes were obtained by a spray pyrolysis technique and then they were decorated by Pt NPs in two different concentrations (5 and $30 \mathrm{wt} . \%$ ). The final compounds, MWCNTs$\mathrm{Pt}_{5}$ and MWCNTs- $\mathrm{Pt}_{30}$, respectively, were suspended in ethanol which is an easy continuous phase of separation. The nanoinks were deposited on a $\mathrm{SiO}_{2}$ matrix in order to maintain the propagation of an electromagnetic wave. The resulting samples presented an average thickness of $1 \mu \mathrm{m}$.

2.2. Morphology Characterization. In order to study the morphology of the metallically decorated nanotubes, two different microcopy techniques were undertaken. The dispersion of the Pt NPs was evaluated by Scanning Electron Microscopy (SEM) using a JEOL JSM-6701F equipment. The multiwall nature of the CNTs was analyzed by Transmission Electron Microscopy (TEM) at $200 \mathrm{kV}$ with a JEOL JEM-2200FS system. To obtain TEM images, a drop of CNTs dispersed in isopropanol was deposited on a carbon coated grid and allowed to dry in air. On the other hand, a transmittance UVvis spectrum experiment was carried out to explore the nanostructures.

2.3. Double-Stage Optical Kerr Gate Experiments. The proposed mechanism to encrypt an optical signal by the phase modification in the polarization was based in a double-stage optical Kerr gate (OKG) with a single source of high energy provided by the second harmonic of Nd:YAG at $532 \mathrm{~nm}$ wavelength. A TEM ${ }_{00}$ Gaussian beam profile at the output of the laser system was employed. Since the observation of nanosecond third-order nonlinear optical effects is dependent on peak irradiances, the shape of the photoinduced laser beams is crucial in the experiments. We observed the complete

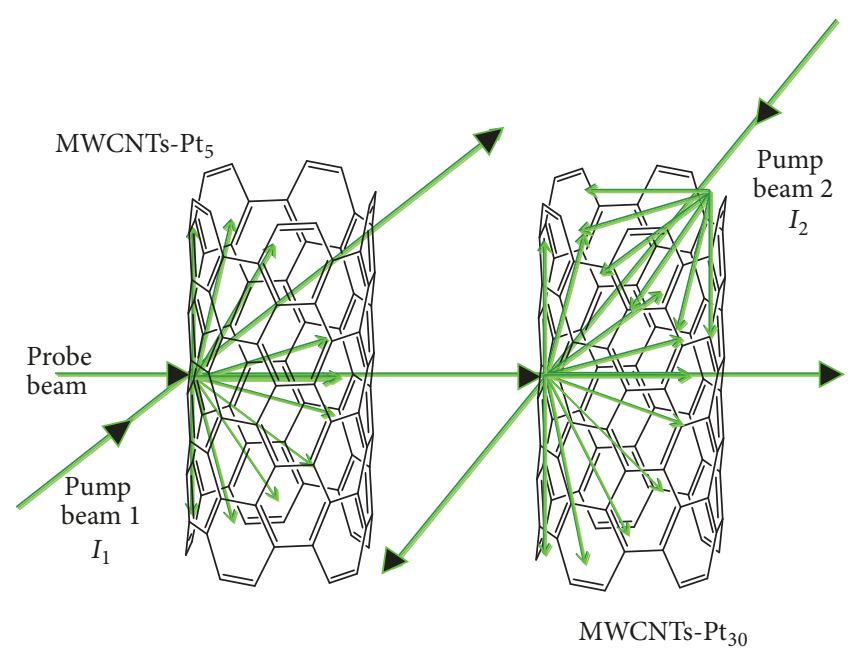

FIgURE 1: Experimental scheme of the double-stage OKG.

inhibition of the Kerr gating by changing the transversal electromagnetic mode of the system in this OKG configuration.

A two-wave mixing (TWM) experiment was conducted to induce a change in the polarization of a probe beam. Probe and pump beams were obtained by splitting a single pulse of $4 \mathrm{~ns}$ duration with linear polarization and a correlation in the energy of $1: 10$. The pump beam, with $24 \mathrm{MW} / \mathrm{cm}^{2}$ irradiance, was retarded in polarization with a half wave plate and oriented at $45^{\circ}$ with respect to the probe, in order to control an OKG. Both beams impinged under the geometric center surface of the samples (MWCNTs- $\mathrm{Pt}_{5}$ and MWCNTs$\mathrm{Pt}_{30}$ ) with a propagation angle of $35^{\circ}$. Opposite changes of optical phase in the samples were measured by rotating the samples from $0^{\circ}$ to $180^{\circ}$. To induce a mechano-optic effect, the samples were placed in a cascade arrangement, the probe beam was propagated through both nanohybrid specimens, and the transmitted light was analyzed by a cross polarizer with respect to the initial polarization of the probe beam. The probe beam irradiance was measured by a high-speed PIN diode. Two modalities were exhibited: the first when the pump beam induced a Kerr effect in the MWCNTs- $\mathrm{Pt}_{5}$ sample and the second when the pump beam excited the MWCNTs- $\mathrm{Pt}_{30}$ film. This mode is obtained when a mirror was displaced linearly to scatter the pump beam into the second sample, as shown in Figure 1.

2.4. XOR Optical Logic Gate Function. A multiphotonic alloptical logic gate based on carbon/metal nanohybrids was based on a double-stage OKG. The system allowed us to rotate the polarization of the transmitted probe beam in each sample as a function on the pump irradiance. The two irradiances $I_{1}$ and $I_{2}$ illustrated in Figure 1 provided the two inputs together to the probe beam as the output to perform a XOR optical logic gate (OLG) device.

\section{Results and Discussions}

Figures 2(a) and 2(b) depict the SEM images of the studied nanohybrid materials. Pt NPs are observed as white points 

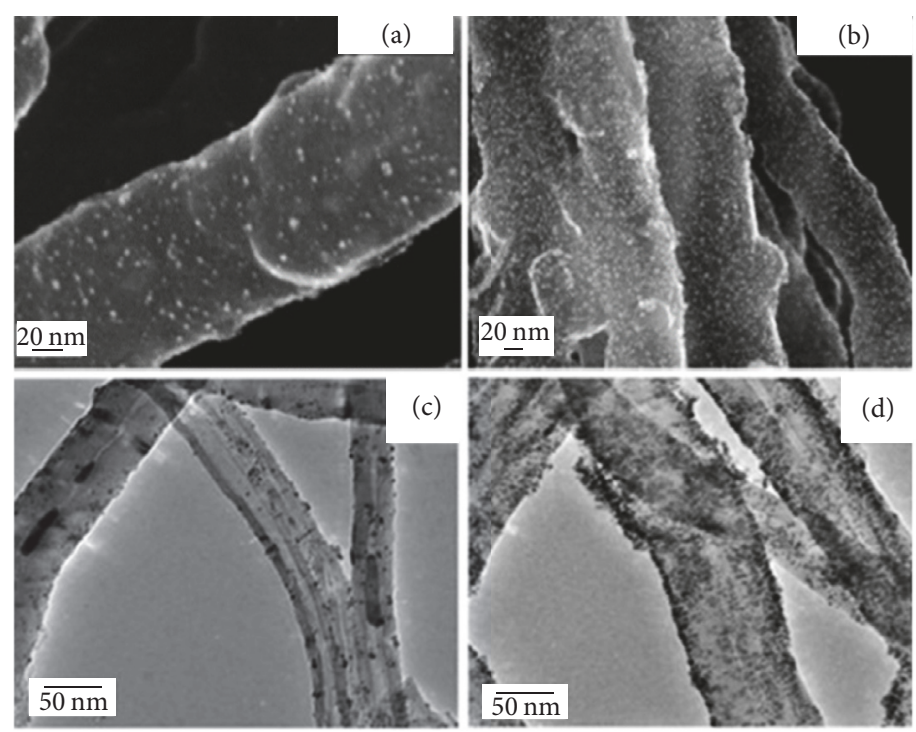

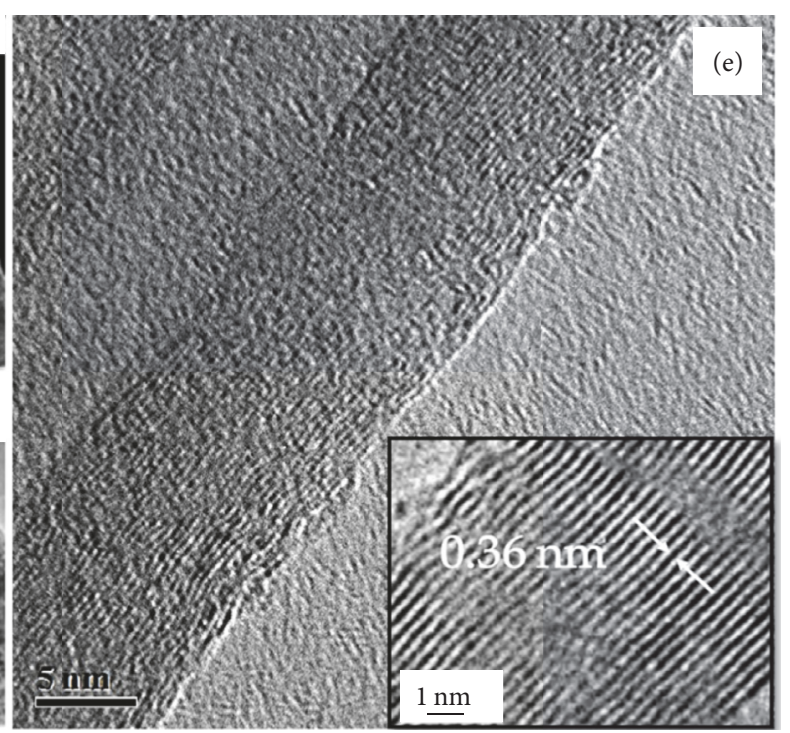

Figure 2: SEM image of MWCNTs with (a) Pt at $5 \mathrm{wt} \%$ and (b) Pt at $30 \mathrm{wt} \%$. TEM image of MWCNTs with (c) Pt at 5 wt\% and (d) Pt at $30 \mathrm{wt} \%$. (e) In HRTEM image of an isolated CNT, the inset shows its multiwall nature.

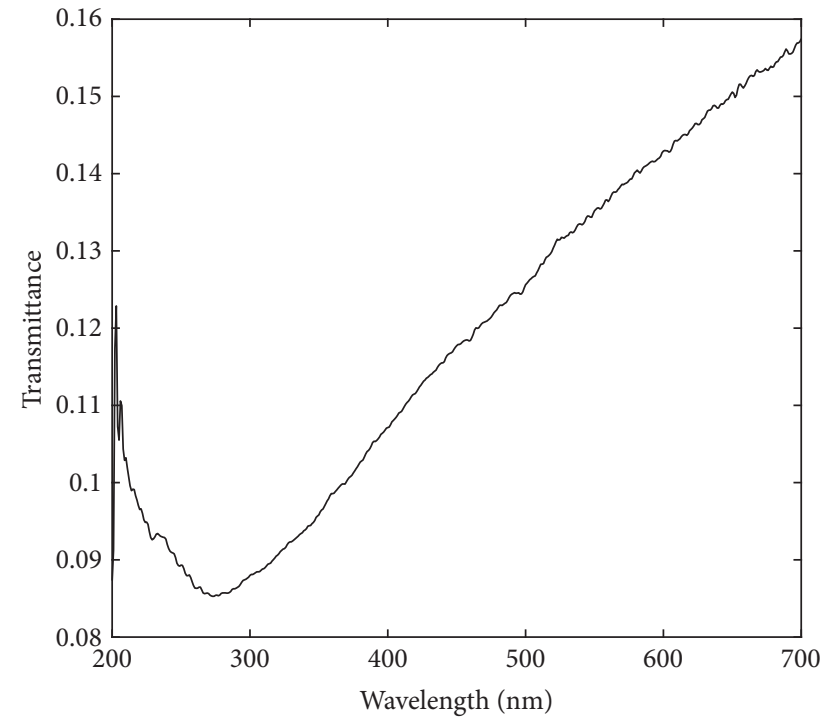

FIGURE 3: Transmittance UV-vis spectrum.

on the nanotubes surfaces. The bigger concentration is clearly observed in the (b) inset. TEM images of Pt incorporation on MWCNTs are presented in Figures 2(c) and 2(d), and these images correspond to different platinum loading: (c) $5 \mathrm{wt} \%$ and (d) $30 \mathrm{wt} \%$. Metal phase is clearly observed as dark dots dispersed on carbon nanotubes surfaces. Furthermore, the multiwall nature of the tubes can be observed in Figure 2(e). The separation between the layers of the graphene rolling sheets was around $0.36 \mathrm{~nm}$.

In order to characterize the optical response of the samples as a function of the wavelength, their UV-vis spectra were analyzed. The typical linear optical transmittance measurements are plotted in Figure 3. A single curve is shown since no differences between the MWCNTs- $\mathrm{Pt}_{5}$ and MWCNTs$\mathrm{Pt}_{30}$ samples were optically identified. The valley observed at
$270 \mathrm{~nm}$ wavelength can be associated with the $\pi-\pi$ resonance exhibited by the CNTs. Apparently, the contribution of Pt to the transmittance spectrum exhibited by the hybrid materials is weak taking into account the volume fraction employed to observe important changes in the nonlinear optical response exhibited by the samples. The transmittance magnitudes correspond to dark samples rather than only allow the propagation of about $13 \%$ of the irradiance at the output of the system for a $532 \mathrm{~nm}$ wavelength. Absorption is mainly responsible for the attenuation of electromagnetic waves close to the resonance valley at the ultraviolet wavelengths; however, the monotonic decrease in the optical transmittance from the optical region to the ultraviolet wavelengths can be considered to be related to scattering effects.

The nonlinear optical behavior in the interaction of the probe and pump beams in the TWM experiment can be explained by solving the wave equation [19]. We used the finite-difference method to numerically approach it.

$$
\nabla^{2} E_{ \pm}=-\frac{n_{ \pm}^{2} \omega^{2}}{c^{2}} E_{ \pm},
$$

where the right $\left(E_{+}\right)$and left $\left(E_{-}\right)$circular components of the electric field can be described by using the Jones matrices. The optical frequency is denoted by $\omega$ and the speed of light in vacuum by $c$. The approximation we take account of for the refractive index was

$$
n_{ \pm}^{2}=n_{0}^{2}+4 \pi\left(A\left|E_{ \pm}\right|^{2}+(A+B)\left|E_{\mp}\right|^{2}\right),
$$

where $n_{0}$ is the linear refractive index, $A=\chi_{1122}{ }^{(3)}$, and $B=$ $\chi_{1212}{ }^{(3)}$ which are elements of the third-order susceptibility tensor $\chi^{(3)}$.

The experimental data of the double-stage mechanooptical modulation is shown in Figure 4. Two sets of measurements are presented for each sample when they are rotating from $0^{\circ}$ to $180^{\circ}$. Both results represented the transmitted 


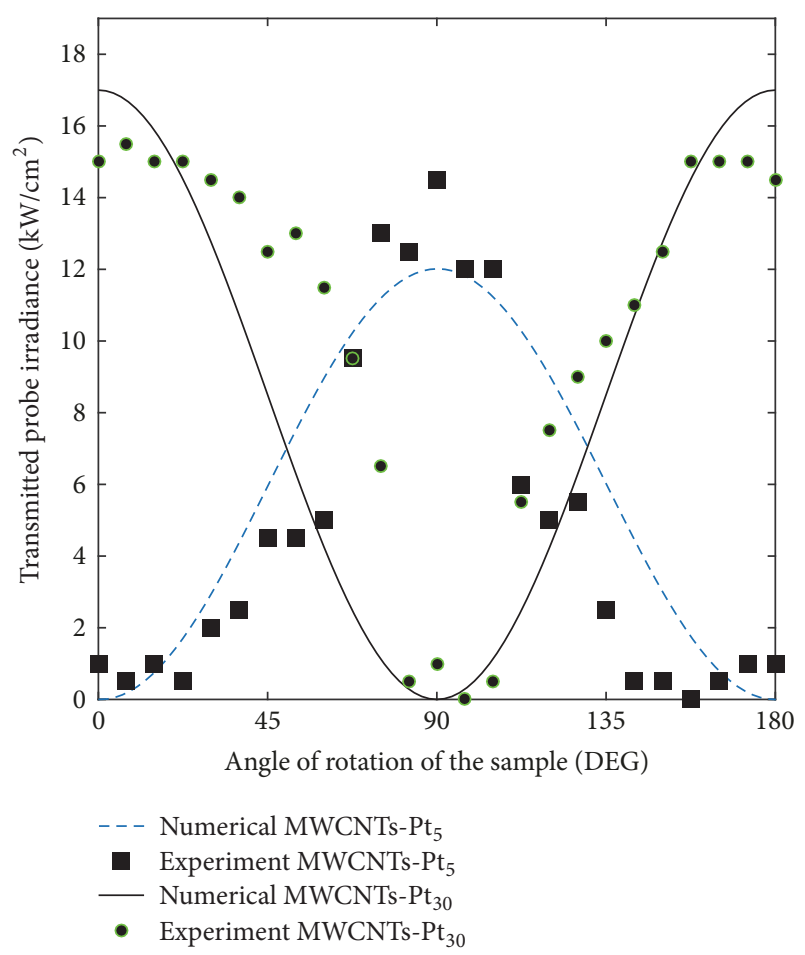

FIGURE 4: Transmitted probe irradiance in the double-stage OKG with rotating samples.

irradiance of the probe beam when the OKG was switching. We considered the minimum Kerr transmittance in the MWCNTs- $\mathrm{Pt}_{5}$ film as the initial point of the stage. In contrast, the maximum value from the complement sample was fixed at the origin. Following this methodology can be considered a binary system, with pump beams as input control signals that encrypt the probe signal. Thermo-optical properties in this set of studied nanostructures have been previously explored by using comparative samples [20]. The contribution of photothermal actions can be controlled in the nonlinear optical signals according to thermal diffusivity, thermal conductivity, and specific heat of the nanostructures. These parameters are also related to the density and morphology of the films together to the energy transfer mechanisms of optical absorption that are also responsible for inducing phononic phenomena. Besides the fact that photothermal effects represent a prevailing influence for nonlinear optical absorption exhibited by carbon/metal samples irradiated by a high repetition rate of laser pulses, significant optical Kerr measurements can be obtained in an OKG configuration by photonic excitations in a single-shot mode.

In Figure 5(a) is shown the positive contribution to the nonlinear polarization of the MWCNTs- $\mathrm{Pt}_{5}$ sample when the pump irradiance was rising. Additionally, the MWCNTs- $\mathrm{Pt}_{30}$ sample presents a negative rotation in the polarization at the same conditions. Regarding this behavior, an exclusive XOR OLG is achieved by placing the two samples in tandem as illustrated in Figure 1. Additionally, Figure 5(b) describes the modification in the angle of polarization of the probe beam interacting with $I_{1}$ and $I_{2}$ and considering the rotation of the samples. The error bar for the optical phase changes that
TABLE 1: XOR logic gate results.

\begin{tabular}{ccc}
\hline $\begin{array}{c}\text { Logic value of the optical beams } \\
I_{1}\end{array}$ & $\begin{array}{c}\text { Transmitted probe beam } \\
{\left[\mathrm{kW} / \mathrm{cm}^{2}\right]}\end{array}$ \\
\hline 0 & $I_{2}$ & 0 \\
0 & 0 & 15 \\
1 & 1 & 14.5 \\
1 & 0 & 0 \\
\hline
\end{tabular}

correspond to the switching of the photoinduced beams is approximately $\pm 12 \%$ in the XOR system.

Table 1 shows the experimental results related to the XOR system designed by double-stage OKG.

The opposite rotation in the polarization of the probe beam was calibrated by exploring the inhomogeneity of the samples in rotation. The experimental analysis of incident irradiance of the different pump beams $\left(I_{1}\right.$ and $\left.I_{2}\right)$ showed a way to build an OLG. Moreover, the MWCNTs samples are easy to fabricate due to the ink treatment of the nanocomposites. The cascade OKG system has the property to encrypt nonlinear information that can be associated with the polarization of the interacting waves [21]. The remarkable third-order susceptibility exhibited by the CNT composites makes them good candidates for encrypting and decrypting photonic information in a tandem of two doublestage arrangements. The nonlinear optical effects exhibited by the nanostructures pointed out their potential to read high energy quantum data [22]. Regarding the influence of hydrostatic pressure and temperature on absorptive optical nonlinearities, phonon anharmonicities could play a key role in the development of irradiance dependent switching carbon-based nanodevices [23]. The third-order nonlinear phenomena exhibited by the samples open the possibility for developing multiphotonic devices and quantum computing [24]. The two-stage scheme proposed in this work has remarkable characteristics to construct OLGs circuits based on mechano-optical modulation effects [25].

\section{Conclusions}

The anisotropic optical Kerr effect exhibited by MWCNTs decorated with Pt NPs was analyzed. The samples were initially prepared as nanoinks and then integrated in thin film form to be explored in a double-stage system by nanosecond OKG experiments at $532 \mathrm{~nm}$. Separate excitation of the third-order nonlinear optical response exhibited by two different samples enables the possibility of delaying the different components of polarization of optical signals. The modulation of the polarization of the photoinduced beams in a TWM experiment was analyzed as a function of mechanooptical and gyroscopic conditions of the nanostructures in rotation. A strong dependence on polarization of the transmitted beams through the samples allowed us to systematically control nonlinear optical signals for photonic applications. Within this work was demonstrated the possibility of tailoring polarization-selectable effects in carbon-based nanostructures by Pt NPs decoration. The collective behavior 


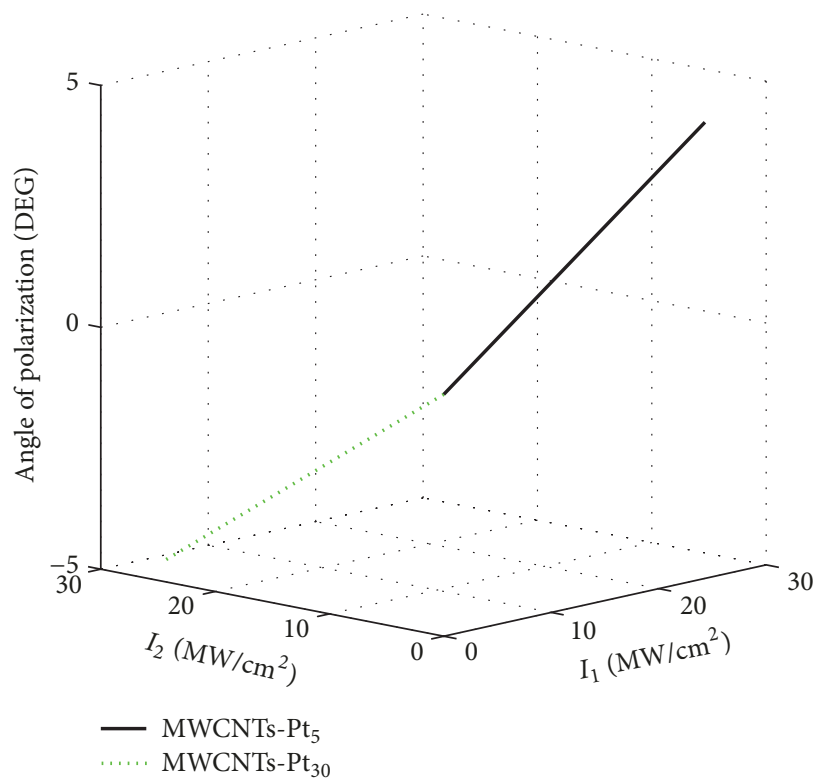

(a)

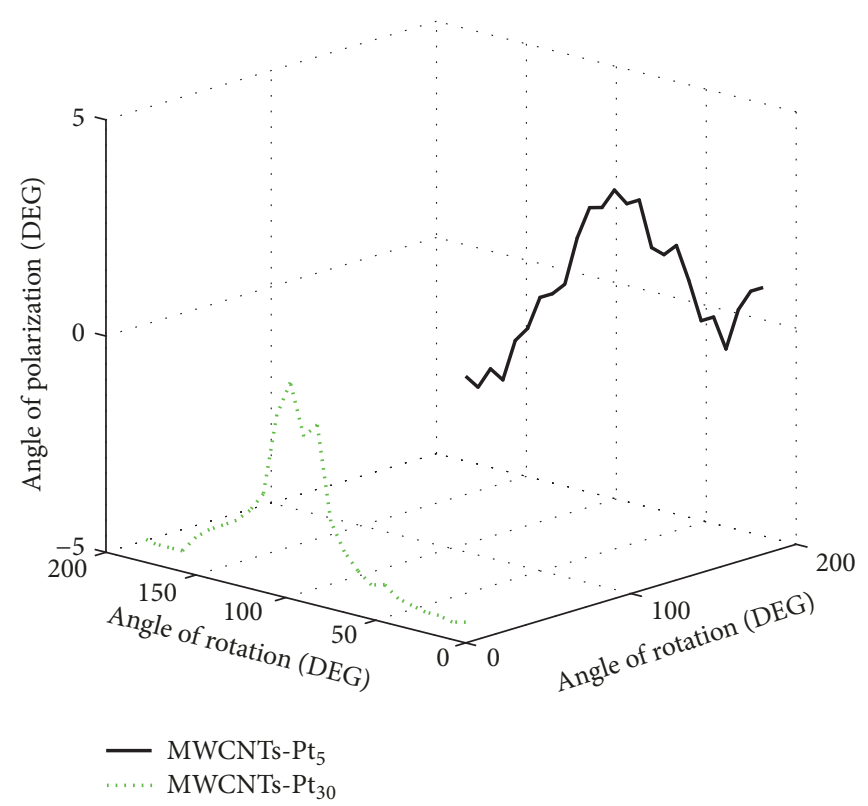

(b)

Figure 5: (a) Angle of polarization versus irradiance of the control beams. (b) Angle of polarization versus angle of rotation of the samples.

of the studied nanosystems presents potential applications to perform all-optical encryption by ultrafast XOR logic functions. In regard to the remarkable nanoscale third-order nonlinear optical effects exhibited by carbon/metal nanoinkbased materials, the design of optical platforms fabricated by advanced nanoinkjet systems can be adequate.

\section{Data Availability}

The authors declare that the data can be obtained upon request by an email to the corresponding author.

\section{Conflicts of Interest}

The authors declare that there are no conflicts of interest regarding the publication of this paper.

\section{Acknowledgments}

The authors kindly acknowledge the financial support from the Instituto Politécnico Nacional, Tecnológico de Estudios Superiores de Coacalco and from the Consejo Nacional de Ciencia y Tecnología (243427 and CB-2015-251201). They also kindly acknowledge the Centro de Nanociencias y Micro y Nanotecnología from Instituto Politécnico Nacional.

\section{References}

[1] S. K. Srivastava and V. Mittal, Hybrid Nanomaterials: Advances in Energy, Environment and Polymer Nanocomposites, John Wiley \& Sons, 2017.

[2] J. d. Ku-Herrera, V. La Saponara, and F. Avilés, "Selective damage sensing in multiscale hierarchical composites by tailoring the location of carbon nanotubes," Journal of Intelligent Material Systems and Structures, vol. 29, no. 4, pp. 553-562, 2017.
[3] I. Di Bernardo, G. Avvisati, C. Chen et al., "Topology and doping effects in three-dimensional nanoporous graphene," Carbon, vol. 131, pp. 258-265, 2018.

[4] R. Vajtai, Ed., Springer Handbook of Nanomaterials, SpringerVerlag, New York, NY, USA, 2013.

[5] W. Hu, B. Zhu, Y. Cao et al., "Preparation and investigation on third-order nonlinear optical properties of ZnS/MWCNTs composite materials," Materials Letters, vol. 172, pp. 44-47, 2016.

[6] J. A. García-Merino, C. L. Martínez-González, C. R. T.-S. Miguel, M. Trejo-Valdez, H. Martínez-Gutiérrez, and C. TorresTorres, "Photothermal, photoconductive and nonlinear optical effects induced by nanosecond pulse irradiation in multiwall carbon nanotubes," Materials Science and Engineering: $B$ Advanced Functional Solid-State Materials, no. B 24, pp. 1955219557, 2016.

[7] H. I. Elim, W. Ji, G. H. Ma, K. Y. Lim, C. H. Sow, and C. H. A. Huan, "Ultrafast absorptive and refractive nonlinearities in multiwalled carbon nanotube films," Applied Physics Letters, vol. 85, no. 10, pp. 1799-1801, 2004.

[8] R. Paul, A. Maity, A. Mitra, P. Kumbhakar, and A. K. Mitra, "Synthesis and study of optical and electrical characteristics of a hybrid structure of single wall carbon nanotubes and silver nanoparticles," Journal of Nanoparticle Research, vol. 13, no. 11, pp. 5749-5757, 2011.

[9] C. M. Hoang, T. Iida, L. T. Dat, H. T. Huy, and N. D. Vy, “Optimal coating thickness for enhancement of optical effects in optical multilayer-based metrologies," Optics Communications, vol. 403, pp. 150-154, 2017.

[10] C. Lertvachirapaiboon, A. Baba, S. Ekgasit, K. Shinbo, K. Kato, and F. Kaneko, "Transmission surface plasmon resonance imaging of silver nanoprisms enhanced propagating surface plasmon resonance on a metallic grating structure," Sensors and Actuators B: Chemical, vol. 249, pp. 39-43, 2017.

[11] C. Lee, E. Sim, and D. Kim, "Effect of Nanogap-Based LightMatter Colocalization on the Surface Plasmon Resonance 
Detection," Journal of Lightwave Technology, vol. 35, no. 21, Article ID 8030981, pp. 4721-4727, 2017.

[12] B. Unal, M. Senel, A. Baykal, and H. Sözeri, "Multiwall-carbon nanotube/cobalt ferrite hybrid: Synthesis, magnetic and conductivity characterization," Current Applied Physics, vol. 13, no. 7, pp. 1404-1412, 2013.

[13] K. Yoshida, S. Arai, Y. Sasaki, and N. Tanaka, "Catalytic oxidation of carbon nanotubes with noble metal nanoparticles," Micron, vol. 76, pp. 19-22, 2015.

[14] Z. He, J. Chen, D. Liu, H. Tang, W. Deng, and Y. Kuang, "Deposition and electrocatalytic properties of platinum nanoparticals on carbon nanotubes for methanol electrooxidation," Materials Chemistry and Physics, vol. 85, no. 2-3, pp. 396-401, 2004.

[15] E. Garrido, C. Aymonier, L. Roiban et al., "Noble metals supported on carbon nanotubes using supercritical fluids for the preparation of composite materials: A look at the interface," The Journal of Supercritical Fluids, vol. 101, pp. 110-116, 2015.

[16] T. Yuan and W. Wang, "Studying the electrochemistry of single nanoparticles with surface plasmon resonance microscopy," Current Opinion in Electrochemistry, vol. 6, no. 1, pp. 17-22, 2017.

[17] C. Gupta, P. H. Maheshwari, and S. R. Dhakate, "Development of multiwalled carbon nanotubes platinum nanocomposite as efficient PEM fuel cell catalyst," Materials for Renewable and Sustainable Energy, vol. 5, pp. 1-11, 2016.

[18] C. Mercado-Zúñiga, J. R. Vargas-García, F. Cervantes-Sodi, M. Trejo-Valdez, R. Torres-Martínez, and C. Torres-Torres, "Photoconductive logic gate based on platinum decorated carbon nanotubes," Applied Optics, vol. 52, no. 22, pp. E22-E27, 2013.

[19] R. W. Boyd, Nonlinear Optics, Academic Press, Amsterdam, The Netherlands, 3rd edition, 2008.

[20] J. A. García-Merino, C. Mercado-Zúñiga, C. L. MartínezGonzález, C. R. Torres-SanMiguel, J. R. Vargas-García, and C. Torres-Torres, "Magneto-conductive encryption assisted by third-order nonlinear optical effects in carbon/metal nanohybrids," Materials Research Express, vol. 4, no. 3, Article ID 035601, 2017.

[21] S. Puri, S. Boutin, and A. Blais, "Engineering the quantum states of light in a Kerr-nonlinear resonator by two-photon driving," npj Quantum Information, vol. 3, pp. 1-7, 2017.

[22] T. Uda, A. Ishii, and Y. K. Kato, "Single Carbon Nanotubes as Ultrasmall All-Optical Memories," ACS Photonics, vol. 5, no. 2, pp. 559-565, 2017.

[23] P. Hudhomme, I. V. Kityk, and X. N. Phu, "Influence of hydrostatic pressure and temperature on two-photon absorption of a (formula presented)-2-thioxo-1,3-dithiole cycloadduct," Physical Review B: Condensed Matter and Materials Physics, vol. 59, no. 14, pp. 9229-9238, 1999.

[24] M.-X. Luo, H.-R. Li, and H. Lai, "Quantum computation based on photonic systems with two degrees of freedom assisted by the weak cross-Kerr nonlinearity,' Scientific Reports, vol. 6, Article ID 29939, pp. 1-15, 2016.

[25] X. Yang, X. Hu, H. Yang, and Q. Gong, "Ultracompact all-optical logic gates based on nonlinear plasmonic nanocavities," Journal of Nanophotonics, vol. 6, no. 1, pp. 365-376, 2017. 

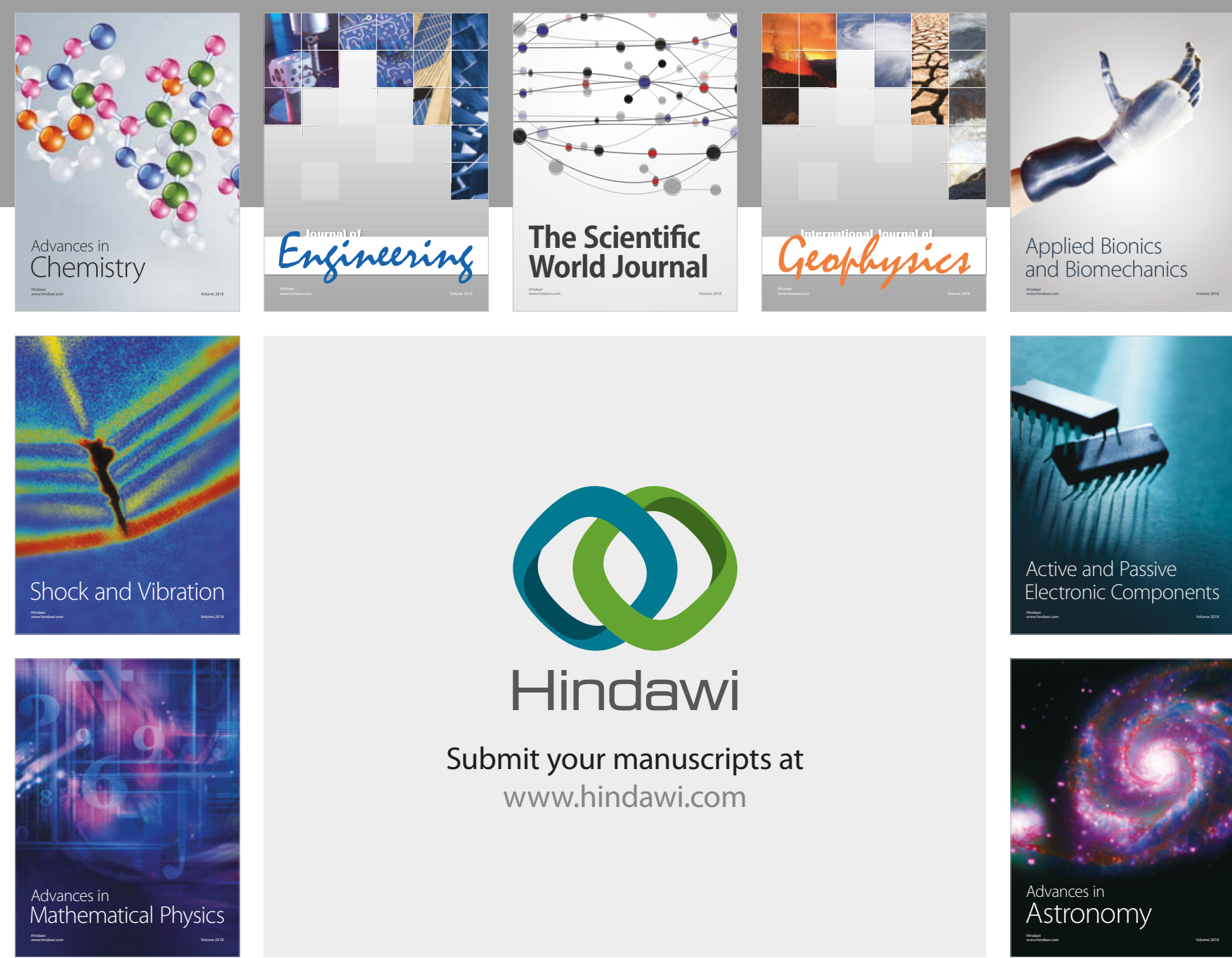

Submit your manuscripts at

www.hindawi.com

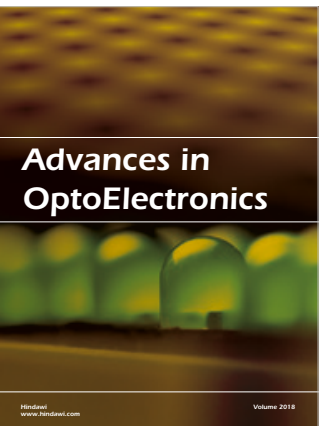

\section{Rotcting Machinery}
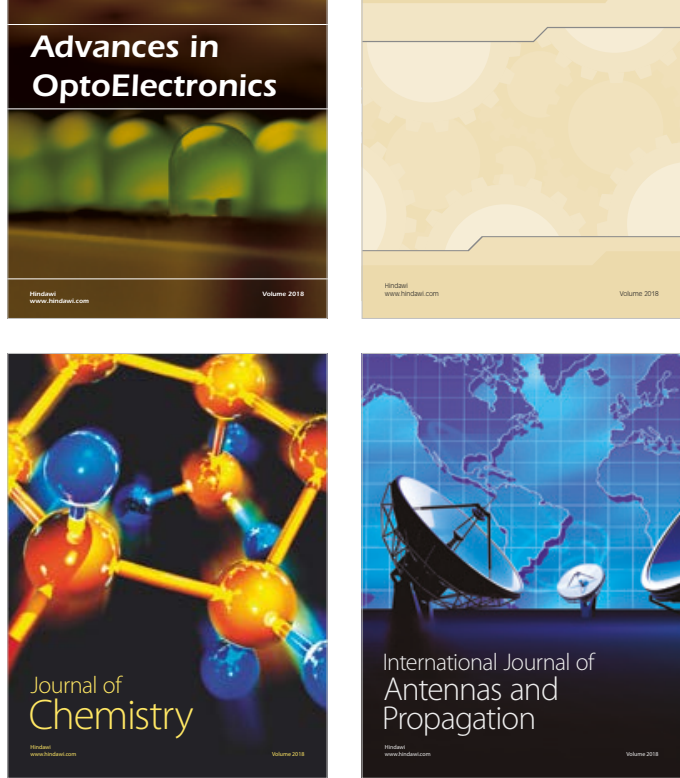

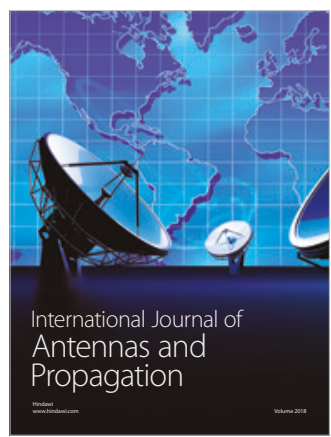

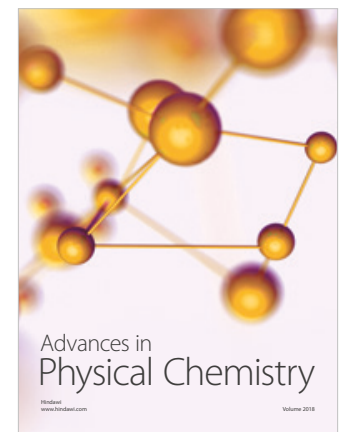

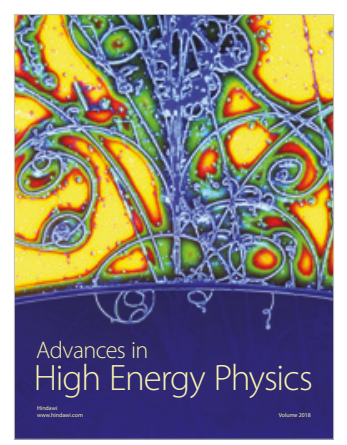

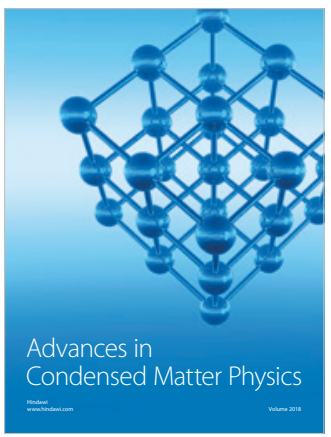

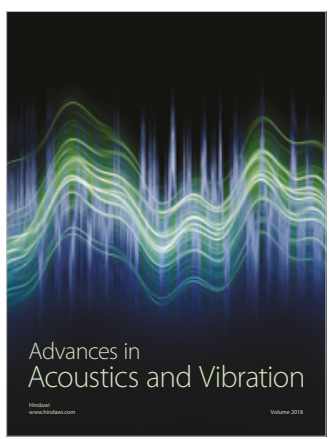

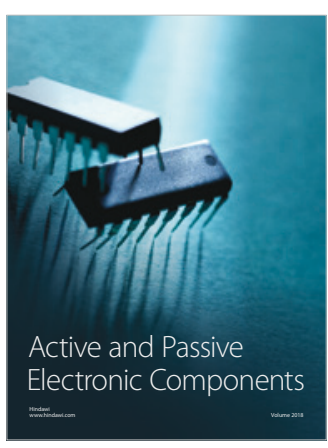
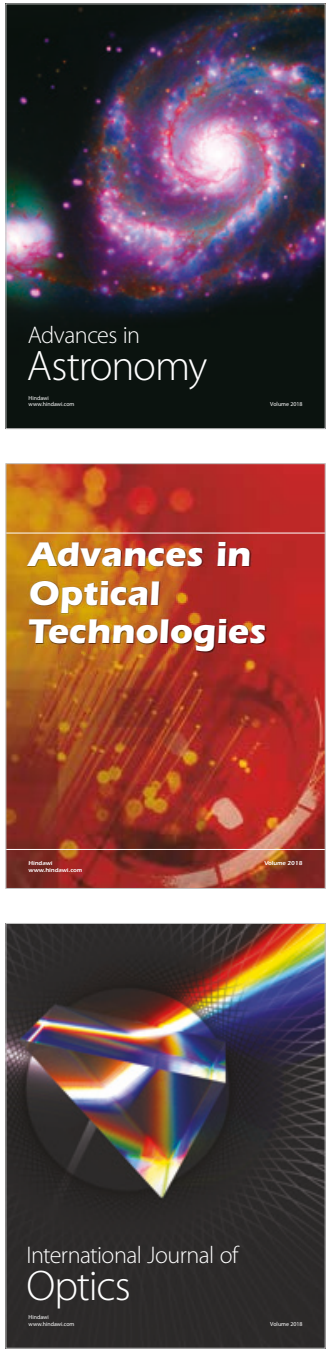